\title{
THE LOG-MINKOWSKI INEQUALITIES FOR QUERMASSINTEGRALS
}

\author{
Wei WANG AND Ming Feng
}

Abstract. Recently, Stancu established the log-Minkowski inequality for non-symmetric convex bodies. In this article, we establish the log-Minkowski inequality for quermassintegrals, which is more general than Stancu's results.

Mathematics subject classification (2010): 52A40, 53A15.

Keywords and phrases: Star body, radial function, dual mixed volumes, dual Minkowski inequality, log-Minkowski inequality.

\section{REFERENCES}

[1] K. J. BÖRÖCZKY, E. Lutwak, D. YANG, G. ZhAng, The log-Brunn-Minkowski inequality, Adv. Math. 231 (2012), 1974-1997.

[2] T. M. Cover, J. A. Thomas, Elements of Information Theory, second edition, Wiley-Interscience, Hoboken, NJ, 2006.

[3] R. J. Gardner, Geometric Tomography, second ed., Cambridge Univ. Press, NewYork, 2006.

[4] R. J. Gardner, D. Hug, W. WeIL, The Orlicz-Brunn-Minkowski theory: a general framework, additions, and inequalities, J. Differential Geom. 97 (2014), 427-476.

[5] R. J. Gardner, D. Hug, W. Weil, And D. Ye, The dual Orlicz-Brunn-Minkowski theory, J. Math. Anal. Appl. 430 (2015), 810-829.

[6] P. M. Gill, C. E. M. PeARCE, J. PeČARIĆ, Hadamard's inequality for $r$-convex functions, J. Math. Anal. Appl. 215 (1997), 461-470.

[7] P. M. GRUBer, Convex and Discrete Geometry, Grundlehren Math. Wiss., vol. 336, Springer, Berlin, 2007.

[8] P. Guan, L. Ni, Entropy and a convergence theorem for Gauss curvature flow in high dimension, preprint, arXiv:1306.0625, 2013.

[9] G. H. Hardy, J. E. Littlewood and G. Pólya, Inequalities, second ed., Cambridge University Press, Cambridge, 1988.

[10] E. LutwaK, Dual mixed volumes, Pac. J. Math. 58 (1975), 531-538.

[11] E. LUTwaK, The Brunn-Minkowski-Firey theory I: mixed volumes and the Minkowski problem, J. Differential Geom. 38 (1993), 131-150.

[12] L. MA, A new proof of log-Brunn-Minkowski inequality, Geom. Dedicata 177 (2015), 75-82.

[13] C. Saroglou, Remarks on the conjectured log-Brunn-Minkowski inequality, Geom. Dedicata 177 (2015), 353-365.

[14] R. SchneIder, Convex Bodies: The Brunn-Minkowski Theory, second ed., Cambridge University Press, Cambridge, 2014.

[15] A. Stancu, The logarithmic Minkowski inequality for non-symmetric convex bodies, Adv. Appl. Math. 73 (2016), 43-58.

[16] W. WAnG, L. J. LiU, The dual log-Brunn-Minkowski inequality, Taiwanese J. Math. 20 (2016), 909919. 